Wroctaw

\title{
LUDWIG WACHLER ALS HISTORIKER DER DEUTSCHEN UND EUROPÄISCHEN NATIONALLITERATUR ${ }^{1}$
}

ABSTRACT: Ludwig Wachler as a historian of the German and European national literature

The article revolves around the research activity of the historian Ludwig Wachler, professor at the University of Wrockaw in the years 1815-1838. In his work, Wachler represents the early German patriotism, for which he was subject to police repression. He is shown primarily as a historian of national literature.

KEY WORDS: national literature, common literature, reaction, political repression in Wrocław in the Biedermeier period

\section{Einige Bemerkungen zum Werdegang Ludwig Wachlers als Vermittler der deutschen Literaturgeschichte an der Universität Breslau}

Der Werdegang des „Litteraturhistorikers“ (d.h. Wissenschaftshistorikers) Ludwig Wachler (geb. 1767 in Gotha, gest. 1838 in Breslau) ${ }^{2}$ wurde bereits würdigend beschrieben. Auch seine Ansichten zur Methodik der Geschichte und zur Pädagogik waren bereits Gegenstand einer eingehenden Untersuchung (Löffler, 2011). In der vorliegenden Betrachtung konzentriere ich mich auf diejenige Rolle, die Wachler nicht als Forscher, sondern eher als Popularisator der deutschen schöngeistigen Literatur (der deutschen Nationalliteratur) auf seinem Breslauer Lehrstuhl von 1815 bis 1838 spielte.

\footnotetext{
${ }^{1}$ Der Aufsatz entstand im Rahmen des Forschungsprojektes UMO-2011/03/B/HS2/04000, finanziert durch das Narodowe Centrum Nauki Rzeczypospolitej Polskiej (Nationales Zentrum für Wissenschaft der Republik Polen). Der Verf. bedankt sich bei der Humboldt-Stiftung, die ihm es ermöglichte, im Sommer 2016 Forschungen zu diesem Thema in den Berliner Archiven und Bibliotheken durchzuführen.

${ }^{2}$ Wachler, A. (1838); Johann Friedrich Ludwig Wachler (1840). Siehe auch eine kurz gefasste Autobiographie bei Wachler (1812, S. 364-376).
} 
Grundlegend sind für mich zwei Werke Wachlers: Vorlesungen über die Geschichte der deutschen Nationallitteratur (Teil 1: Frankfurt am Main 1818, Verlag der Hermannschen Buchhandlung; Teil 2: 1819) sowie sein Handbuch der Geschichte der neueren Literatur (Teil 1: Frankfurt am Main 1824, Verlag der Hermannschen Buchhandlung). Flankiert wird die vorliegende Analyse durch die Heranziehung bisher nicht ausgewerteter Archivmaterialien aus dem Hessischen Staatsarchiv Marburg, in dem sich der Nachlass Wachlers befindet sowie des in der Breslauer Universitätsbibliothek aufbewahrten Briefwechsels Wachlers mit diversen Teilnehmern wissenschaftlicher Kommunikation.

Wenn man die spezifische Position Wachlers reflektiert, dann muss man nicht nur auf seine ideologisch bestimmte Akzeptanz des Nationalen Acht geben, sondern auch seine Vorstellungen zur eigenen Rolle als Professor heranziehen, wozu er sich bereits als Hochschullehrer in Marburg geäußert hatte (Wachler, 1802). Die Hauptaufgabe des Vortrages ist ,den Zuhörer zum praktischen Geschäftsmann durch wissenschaftliche Theorie zu bilden“ (ebd., S. 87). Der Lehrer hat im Rahmen seines „deutlichen“ und klaren Vortrages einerseits die „wichtigern Veränderungen, welche die vorgetragene Wissenschaft in neueren Zeiten erlitten hat" (ebd., S. 89), darzubieten, aber andererseits auch „den Standort, von dem aus die Gestalt der Wissenschaft gewürdigt werden kann, bemerklich zu machen“ (ebd.).

Die Bestallung Wachlers nach Breslau erfolgte Anfang 1815. Am 19. Februar informierte der preußische Innenminister von Schuckmann die Academische Verwaltungs-Commission über die Entscheidung des Königs, Wachler in Nachfolge des verstorbenen K. F. Bredow als ordentlichen Professor zu installieren ${ }^{3}$, woraufhin Wachler seine Vorschläge für die Vorlesungen darbot und versprach, Anfang Mai in Breslau zu erscheinen. Am 23. Februar hat das Ministerium Wachler und die Akademische Kommission in Breslau darüber informiert ${ }^{4}$. Der Gelehrte bedankte sich beim Ministerium mit folgendem Brief:

Euer Excellenz sage ich ehrerbietigsten Dank für die Mittheilung der Königlichen Bewilligung meiner Anstellung; ich hoffe Kraft und Willen zu haben, um auf eine des mir unbeschreiblich theueren Preußischen Staates würdige Weise mich wirksam zu zeigen. Denn daß es dieser höhere edle Lebensreiz ist, der mich K. Preußische Dienste wünschen ließ, werden Ew. Excellenz ohne meine ausdrückliche Erklärung verstanden haben; mein Gewißen saget mir, daß die meisten Beweggründe meine Neigung leiteten. Alle Vorschläge, um mich hier zurück zu halten, habe ich zurückgewiesen; den wiederholt nachgesuchten Abschied kann ich nicht abwarten, sondern werde mir denselben nachschicken laßen. In den nächsten Tagen reise ich mit meiner Familie ab; bleibe einige Wochen in meiner Vaterstadt Gotha und anderen auf dem Reisewege liegenden Städten und werde noch vor Ende des M. April in Breslau eintreffen. ${ }^{5}$

\footnotetext{
${ }^{3}$ GStAPK IHA Rep 76 Va Sekt 4 Tit X Nr. 1, Bd. 3, Bl. 184 r.

${ }^{4}$ GStAPK IHA Rep 76 Va Sekt 4 Tit X Nr. 1, Bd. 3. B1. 185 r-186 v.

${ }^{5}$ Brief Wachlers vom 9. März 1815 an das Ministerium. GStAPK IHA Rep 76 Va Sekt 4 Tit X Nr. 1, Bd. 3, Bl. 198 r.
} 
Als Bibliothekar an der sehr gut ausgestatteten Breslauer Anstalt und als Historiker hatte Wachler ausgedehnte Kontakte mit Gelehrten, Buchhändlern, Verlegern, wovon sein Briefwechsel ein wohl partielles Zeugnis ablegt. Der von ihm vertretene Standort war patriotisch und demokratisch im Sinne des neuen deutschen Patriotismus, den er mit Friedrich Jahn und dessen Turnbewegung teilte, woraus er auch amtlich und öffentlich keinen Hehl machte. Im Begleitbrief zur Sendung seiner patriotischen Broschüre (Wachler, 1814) an den Staatsminister von Hardenberg betonte er pathetisch:

Wenn Tausende für das Vaterland kämpfen, darf der Einzelne, dem nicht vergönnt ist, die Waffen zu tragen, nicht müssig seyn und nicht versäumen um zur Errettung und Unterhaltung der Gesinnungen mitzuwirken, welche die jetzige Zeit und das Bedürfniß des Vaterlandes erheischen. ${ }^{6}$

Als Professor in Breslau und als Konsistorialrat wurde er vom Ministerium und auch von der Provinzbehörde stets unterstützt. Wachler hatte wohl, neben seiner Verpflichtungen als Bibliothekar, Professor und Mitglied der Prüfungskommission auch die Aufgabe, das Ministerium über die zu berufenden Kandidaten im Bereich der protestantischen Theologie zu informieren. So tat er es im Falle des Professors Klein aus Würzburg.?

In Breslau fand der Gelehrte sehr gute Möglichkeiten für sein Wirken als Literaturhistoriker. Erstens war Breslau diejenige Stadt, von der aus die Initialzündung für die Befreiungskriege ausging. Zweitens fand er an der neuen Universität, an die er 1815 berufen wurde, gute Freunde, die seine patriotischen Meinungen durchaus teilten, wie Johann Gustav Gottlieb Büsching oder Friedrich Heinrich von der Hagen, obwohl er sich von den beiden institutionell distanzierte. Eine Verwandtschaft verband ihn mit der Familie Passow, insbesondere mit dem hervorragenden klassischen Philologen und Anhänger der Turnbewegung Franz Passow (L. Wachler, 1835, S. 329; A. Wachler, 1839) ${ }^{8}$. Über die ersten Eindrücke in seiner neuen Heimat, also in Breslau, schreibt er in einem Brief an Professor Johann Samuel Ersch in Halle:

Breslau 17 May XV Da sind wir denn in der neuen Heimath fast 4 Wochen und fangen an, schon ziemlich eingerichtet zu seyn. Es gefällt uns Stadt und Volk ganz wohl; um ein Urtheil abgeben zu dürfen, sind Monate oder Jahre erforderlich. Mit der Bibliothek kann ich für mein Fach zufrieden seyn; ob ich gleich in der Litterarges. vieles vermiße, was ich selbst besaß und in Marburg, um Transportkosten zu ersparen, verkauft habe. Für die polit. u Kirchenges. ist besser gesorgt und die

\footnotetext{
${ }^{6}$ Brief von L. Wachler an den Staatsminister von Hardenberg, Marburg, d. 18 März 1814. Universitätsbibliothek Breslau, Handschriftenabteilung, AKC 1952 KN 9. Der Minister hat sich am 21. März für die Zusendung der Broschüre bedankt.

${ }^{7}$ GStAPK IHA Rep. 76 Sekt 4. Tit IV, Nr. 1, Bd. 5, Bl. 33r. Klein war Philosophie-Professor und ein Schüler Schellings.

${ }^{8}$ Franz Passow (1786-1833), klassischer Philologe, war ein glühender Anhänger des Turnens (Passow, 1818).
} 
Sammlung hat meine Erwartungen weit übertroffen. Den sehr beträchtl., gewiß mit vielem KlosterUnrathe übersättigten, handschriftl. Vorrath will ich in diesen Tagen durchgehen. Meine Antwort aus Gotha auf Ihren Antrag werden Sie richtig erhalten haben. Jetzt ersuche ich Sie ergebenst, die anliegenden Aufträge meinem dortigen Bevollmächtigten zu übergeben und was ich etwa erhalte, mit anderen Breslauer Sachen zusammen, mit möglichst wohlfeiler Gelegenheit uns zukommen zu laßen. Den Geldbetrag will ich Ihnen zu seiner Zeit sogleich in Leipzig anweisen. Empfehlen Sie mich Hr. Hof. Schütz, Canzel. Niemeyer, Pr. Schmaltzer, Gesenius u wer sonst meiner gedenkt. Es that mir Wehe, so nahe bei Gotha zu seyn, als ich von Jena nach Leipzig fuhr, ohne es sehen zu können, aber ich eilte, um dem Ansturme nicht auf der Heerstraße zu begegnen. Und es war die höchste Zeit, als wir den 20ten April hier einzogen, waren die ersten Russen eingerückt. ${ }^{9}$ Mögen uns bald Friede und Ordnung werden, ich fürchte, unser Leben ist zum Sturm und Drang verurtheilt. Nun so möchten die Leute es besser haben. Was macht unser Erz-Marburger Niemeyer? Mit der Versicherung der herzlichsten Ergebenheit Ganz der Ihrige DWachler Herrn Prof. Ersch In Halle Frey[.] ${ }^{10}$

Ludwig Wachler war in erster Linie Historiker und Wissenschaftshistoriker. Kein Archivar und Handschriftenkundler. Bereits 1820 wurde ein Plan zwischen dem neu berufenen Archivar Gustav Adolf Harald Stenzel (vgl. Markgraf, 1892) und Wachler abgeschlossen (Rescript vom 13. Juni 1820 Nr. 7241)

über die geschichtlichen Vorlesungen, sind die diesfälligen Vorträge über die obengenannten Hülfswissenschaften der Geschichte dem Professor Stenzel überweisen worden und da derselbe zugleich Dirigent des schlesischen Provinzial-Archivs ist: so besitzt er einen sehr förderlichen Apparat zu diesen Vorlesungen. ${ }^{11}$

Um seine Stellung und politische Ansichten noch deutlicher zu umreißen, bespreche ich kurz anhand von Archivmaterialien die Konsequenzen des sogenannten Turnstreites (1819) für ihn sowie die Ergebnisse einer polizeilichen Beobachtung aus dem Jahr 1822.

\section{Zum Habitus von Ludwig Wachler}

Einige Aufschlüsse über den Habitus des Gelehrten - zum Habitus der Germanisten im 19. und 20. Jh. siehe Rosenberg (2009) - geben seine politischen und programmatischen Schriften sowie die Breslauer Korrespondenz gute Auskünfte. Er war sich seiner untergeordneten Rolle im damaligen Wissenschaftsbetrieb durchaus bewusst, was er mit seiner Wirkung an kleinen Universitäten wie Rinteln oder

\footnotetext{
${ }^{9}$ Menzel schreibt darüber: „Im Verlauf des Sommers und Herbstes kamen durch Breslau viele Russen, die aus Frankreich heimzogen. Sie brachten ungeheuer viel Beute mit. Wir sahen lange Wagenzüge, die den Raub fortführten, und unterschieden kostbare Tapeten, Teppiche, Spiegel, Uhren usw., die gewiß nicht in Paris gekauft waren“ (1877, S. 75 f.).

${ }^{10}$ Universitätsbibliothek Breslau, Handschriftenabteilung, Aut. 1590

${ }^{11}$ Schreiben des Universitätskurators Neumann an das Ministerium vom 14. Sept. 1829. GStAPK IHA Rep. 76 Va Sekt 4. Tit. IV Nr. 1, Bd. 15, Bl. 15 r.
} 
Marburg in Verbindung brachte. Mehr als die öffentliche Kritik seiner Positionen mussten ihn die abwertenden Bemerkungen eines Karl Rosenkranz schmerzen, er sei lediglich ein Bibliograph, was für die erste und zweite Fassung seines Handbuches durchaus gelten konnte. ${ }^{12}$ Noch krasser wird seine ganz andere Sicht bei den Vorlesungen. Erstens fußen Wachlers Erkenntnisse keinesfalls auf den Resultaten eigener philologischer Arbeit, vom Standpunkt der damaligen wissenschaftlichen Fraktion (Jacob Grimm, Moritz Haupt, vor allem Karl Lachmann) war er also ein Dilettant und konnte kaum die wissenschaftliche Haltung repräsentieren ${ }^{13}$, weil er keine speziellen Untersuchungen vorgelegt hatte. Andererseits muss man gerade die Meinung revidieren, dass die Erzählung der deutschen Literaturgeschichte erst durch Karl Rosenkranz die engen Schranken des von der wissenschaftlichen Fraktion gewünschten Profils sprengte. Bei Wachler werden die drei Elemente durchaus ausgeprägt und zwar mit Bezug auf die Philosophie der Romantik und die Ideologie des neuen Patriotismus, die im Volk das Ziel der wissenschaftlichen Bestrebungen wahrzunehmen glaubte. Man sieht bei ihm das Überspringen der Disziplingrenzen, den deutlichen Bezug auf die Moderne (siehe oben seine Standpunktbestimmung) und vor allem die Orientierung auf eine breitere Öffentlichkeit ${ }^{14}$, der er sehr viele Zuhörer in den Jahren von 1815 bis 1838 verdankte.

Die Breslauer Turnfehde war eine Auflehnung der Schüler am Elisabetanum im Namen der Ideologie des deutschen Patriotismus, die in den Turnerschriften von Friedrich Jahn geprägt wurde. Es haben sich sofort zwei Lager auch unter den Professoren gebildet, die sich pro und kontra aufsässige Jugend erklärten. Die tolerante Haltung Wachlers gefiel dem Ministerium deshalb nicht, weil er als Consistorialrat das Benehmen der Schüler überwachen sollte.

Die von Eurer Excellenz über das Benehmen und die in Druckschriften oder Briefen geäußerten thadelnswerthen Grundsätzen der Consistorialräthe und Professoren Gass ${ }^{15}$ und Wachler, des Professors Passow in Breslau, mittels der in solchen Schreiben am 11ten und 24ten Juny d. J. ingleichen vom 17ten v. Wert. mir gemachten Mittheilungen habe ich um so mehr aufs sorgfälltigste geprüft, als die Stellungen gedachter Männer wichtig und einflußreich sind und es so sehr zu wünschen ist, daß in die schon so lange bewegten und zerrütteten Verhältnisse unter den Breslauer Universitätslehreren und Schulmännern, Ruhe und Friede wiederkehren möge. ${ }^{16}$

Anfänglich war der Minister von Altenstein ziemlich gnädig und schickte an Wachler lediglich eine Rüge:

\footnotetext{
${ }^{12}$ Der „wackere Wachler“ wird kritisiert (Rosenkranz, 1832, S. 10), er habe die „orientalische Poesie“ nur „kurz dargestellt“, in Wirklichkeit hat er sie mit einigen Sätzen abgetan.

${ }^{13} \mathrm{Zu}$ dem Ethos der frühen Germanisten siehe: Wägenbauer (2000).

${ }^{14}$ Diese Merkmale wurden von Klaus Weimar erst Karl Rosenkranz zugeschrieben (1989, S. 303).

${ }^{15}$ Joachim Christian Gaß (1766-1831) war protestantischer Theologe und Professor an der Breslauer Universität $(\mathrm{Gaß}, 1878)$.

${ }^{16}$ Schreiben des Regierungsbevollmächtigten (Neumann) an Minister von Altenstein vom 4. November 1824. GStAPK IHA Rep. 76, Sekt 4, Tit IV Nr. 5. Bl. 16 v.
} 
Aus der über das dortige Turnwesen angestellten Untersuchung geht zwar hervor, daß die gewesenen Schüler des dortigen Elisabeth Gymnasio Haake Menzel ${ }^{17}$ und Lindenberg welche diese Anstalt aus Trotz und Ungehorsam gegen ihre Lehrer verlaßen hatten, bei Ihnen nicht die günstige Aufnahme gefunden haben, welche in dem über diesen Vorfall von dem eben genannten Menzel verfaßten und höchst tadelnswerten Libell ein Beweis ihrer Vorlieben für die Sache des Turnens gerühmt wird. Aber auf der anderen Seite ist auch nicht zu verkennen, daß Sie in dieser Angelegenheit nicht ganz unbefangen gehandelt, vielmehr den Klagen und Beschwerden der gedachten Schüler, ohne deren Grund oder Ungrund näher zu untersuchen und desfalls mit ihren Lehrern in Verhandlung zu treten, Glauben geschenkt, und dadurch wenigstens den Schein einer Partheilichkeit für die Schüler gezeigt haben, was sich mit Ihrer Vorstellung als Konsistorial und Schulrath keinesweges vertrug. ${ }^{18}$

Der Minister Altenstein erwog im Angesichte dieser politischen Vergehen jedoch auch andere Maßnahmen:

3. Schreiben wegen des Professors Wachler. In diesem wird zu äußern seyn, ich würde keine Bedenken getragen haben auf das Schreiben vom 11ten Juny den Antrag auf Entfernung des Wachler von den Consistorial-Raths-Geschäften und seiner Versetzung beyzupflichten, wenn nicht in dem gleichzeitigen Gutachten Sr. Excellenz vom 11 Juny ein Bericht über neuere Aeusserungen des Wachler vorzüglich in Beziehung auf den Professor de Wette ${ }^{19}$ enthalten gewesen seyn. Hiernach pflichte ich vollkommen bey, daß es nicht bloß bei einem Verweiß, und Versetzung verbleiben können, sondern daß ernstlichere Maasregeln erforderlich seyen. Nur rücksichtlich der zu ergreifenden Maasregeln habe ich einiges Bedenken. Eine gerichtliche Untersuchung habe das Unangenehme, daß es die Entscheidung sehr verlängern und die de Wette Angelegenheit zum Gegenstand einer Erörterung vor Gericht mache, welche zumal bey der Ungewißheit des Erfolgs zu umgehen seyn dürfte. Ich halte daher für angemessener, daß der Wachler kommissarisch über diesen Gegenstand im Wege der Disciplin zur Verantwortung gezogen werde und halte die Sache für so angethan, daß gleichzeitig dem Konsistorium davon Nachricht gegeben und solchem eröffnet werden könnte, daß der Wachler sich bis zu erfolgter weiterer Entscheidung aller Theilnahme in den Consistorial Geschäften zu enthalten habe. Hierdurch werde sogleich Ernst gezeigt und der Wachler vorerst unschädlich gemacht. Nach dem Resultate werde sich beurtheilen lassen, ob sich die Sache zur freundlicher gerichteten Untersuchung und Erkenntniß und zur förmlichen Suspension neige, oder ob das Ganze mit dem Grund der allerhöchsten Kabinetts-Ordre vom 12ten April zur Erledigung im Disciplinar-Wege angethan sey. ${ }^{20}$

Die Lage Wachlers verschlimmerte sich zusätzlich aufgrund einer Bespitzelung seines Studenten, in dessen Heft die Spitzel einige vom Standpunkt der Reaktion verwerflichen Bemerkungen zu finden glaubten. Der Regierungsbevollmächtigte hat

\footnotetext{
${ }^{17}$ Der berühmte Kritiker Wolfgang Menzel wird die Breslauer Turnfehde von seinem Gesichtspunkt aus beschreiben (1877, S. 84-150).

${ }^{18}$ Brief des Ministers Altenstein vom 18. Juni 1819. HStaM, Nachlass L. Wachler.

${ }^{19}$ Es geht um eine Äußerung im Dezember-Heft der Theologischen Annalen von 1820. Minister Schuckmann schickte am 11. April eine Anfrage an das Königliche Ober-Censur-Kollegium mit der Anfrage in dieser Sache. Von Altenstein wurde die Sache benutzt, um die Maßnahmen gegen Wachler zu verschärfen. GStAPK IHA Rep. 101 Nr. E Lit. A 06.

${ }^{20}$ Schreiben des Ministers Altenstein an den Innenminister Schuckmann vom 9. Dezember 1822. GStAPK IHA Rep. 76, Sekt 4, Tit IV Nr. 5. Bl. 45 v-46 r.
} 
im Jahr 1824 Wachler ,über einige Stellen seiner literaturgeschichtlichen Vorlesungen aus dem Jahre 1822 " vernommen. ${ }^{21}$

Nun ist mir der ehemalige hiesige Student v Willer seinem Äußeren nach immer fanatisch und verdächtig erschienen, zumalen er als geborener Mecklenburger an der Universität zu Halle als Confilister hierher kam. Demzufolge habe ich ihn auch theils von Seiten der Universität, theils unter Rücksprache mit dem damaligen Polizey-Präsidenten Streit, näher beobachten, ihn sogar persönlich zu mir kommen lassen, ihm Vorstellungen und Warnungen über die Beibehaltung seiner auffälligen deutschen Tracht und über die dadurch entstehende verdächtige Mißfälligkeit ernstlich zu Gemüthe geführt, ja ihm sogar zur Bedingung gemacht, daß er bey akademischen Feierlichkeiten entweder sich anders kleiden, oder, wenn er aus vorgegebener Armuth dies nicht vermöchte, sich ganz hinterwärts halten müßte. (...) Ein Student dieser Art und von so geringer Verstandesbildung und so wenigen Kenntnißen, wie sein Heft an so vielen Stellen beweiset, kann nun wohl wie der p. Wachler behauptet, würklich den Vortrag im Einzelnen oft unrichtig aufgefaßt und, nach seinen vorherrschenden Ansichten umgestaltet, niedergeschrieben haben. (ebd.)

Am Rande sei vermerkt, dass der Bericht Neumanns auch als ein Zeugnis für Wachlers ungeheuere Popularität gelten kann:

Freilich gibt der freie Vortrag des p. Wachler, wie ich theils selbst bemerkt, theils so häufig von anderen gehört, habe, seinen Vorlesungen das lebendige Interesse, was ihm eine so große Frequenz zuzieht, denn uach in dem jetzigen Winter Semester sind wieder seine Vorlesungen so besucht, dass die gewöhnlichen Hörsäle zu klein sind, und daß er daher wieder in dem Großen Musiksaale lesen muß. (ebd., B1. 197 v.)

Wachler wurde noch einmal von Neumann am 2. November 1824 vernommen: er musste sich über einige Stellen verantworten, die im Heft des ehemaligen Studenten Carl von Willer gefunden worden waren. Der Vortrag behandelte die Geschichte der Litteratur der drei letzten Jahrhunderte. Wachler erklärte, daß

auf nachgeschriebene Universitäts Hefte wenig zu vertrauen ist, wie die von Gundling über Litteratur Geschichte, von Marcus über Exegese, von Cant über Metaphysik und Religionslehre anderer zu geschweigen, deutlich berichten. Unvollständigkeit im Materiale führt Mißverständniße herbey, indem oft der Vordersatz unterdrückt und der Nachsatz geschrieben wird, oft auch umgekehrt, dazu kömmt, daß der nachschreibende seiner eigenthümlichen Stimmung nicht entsagt, auch im Collegio nicht, manches verkehrt faßt, manches linkisch ausdrückt. Er selbst, der C.R. u. P. Wachler hat einen völlig freien Vortrag, ohne Hefte: daher er schlechte Hefte weder berichtigen noch buchstäblich widerlegen kann: indem er lediglich darauf ausgeht, die Vollständigkeit zu merken und zum häuslichen Fleiße nachdrücklich anzuhalten. Daß in einem freien Vortrage ein und der andere unedle Ausdruck unterläuft, muß als Mangel anerkannt und wird hoffentlich um so nachsichtiger beurtheilt werden, da sehr vielen würdigen Männern ähnliche Nachläßigkeit verziehen worden ist.

\section{Vom Ministerium wurden folgende 12 Formulierungen Wachlers beanstandet:}

Was die Stelle Seite 17 betrifft, so beziehen sich Lobeserhebungen der Universität Jena hauptsächlich, was mit einem Worte in dem Hefte angedeutet wird, auf die Pflege der Kant- Fichte- Schel-

${ }^{21}$ GStAPK IHA Rep 76 Vf Lit. W. Nr 7 Wachler, Bl. 198. 
ling- und Hegelschen Philosophie. Von dieser Pflege, besonders seit dem Jahre 1784 wo Herr Deponent selbst dort studirt hat, wird die Selbständigkeit des akademischen Lebens abgeleitet, weil es ein wissenschaftliches ist, für welches der Heftschreiber vielleicht gar nicht empfänglich gewesen ist. Was der Ausdruck: Kernleben der dort studierenden Jünglinge betrifft, so findet Herr Deponent, wie auch die dabeygesetzten Jahreszahlen 1812, 1813 zu erkennen geben, darin seine Erläuterung, daß die Studenten von Jena sich zu den preußischen Fahnen hindrängten, um Theil zu nehmen an der Befreiung Deutschlands.

Der Ausdruck: kein verborgenes Gift soll ein Gegensatz gegen andere ... Anstalten, das Nichtvorhandenseyn bösartiger Vergnügungen, Ausschweifungen und Heimlichkeiten seyn.

Das Studentenleben konnte daher geringerer Bewachung bedürftig erscheinen, welches der einzige Sinn seyn kann, wenn in dem Hefte gesagt wird: daß es sich überlaßen sey und ist damit nimmermehr eine ungeziemende Studenten-Freiheit gemeint werden.

$\mathrm{Daß}$ im 18ten Jahrhundert Jena in Bildung von Theologen, Juristen und Medizinern sich vorzüglich wirksam und dadurch segenbringend für alle Provinzen bewiesen habe, wird kaum eines Widerspruchs fähig seyn können.

2. Seite 18 ist die Äußerung über die Lehrfreiheit in Göttingen, wie H.O.R. Wachler bemerkt, lediglich auf das Verhältnis der Universität Göttingen zu England zu bringen, wie die in dem Hefte selbst angeführten Namen von Schmaust - den der unwissende Heftschreiber Schmauz geschrieben hat - und Schlözer, deutlich beweisen. Dabei ist nach der Versicherung des H. O. Wachler, kein Gedanke gewesen, das Verhältniß des akademischen Lehrers zum Staate auch nur einen Augenblick in Zweifel zu stellen.

3. Seite 19-20 erklärt der Herr Prof. Wachler: daß aus den unzusammenhängenden Worten, von der 2ten Zeile Pag. 19 von unten an, kein vernünftiger Sinn herausgebracht werden kann, indem ein Sprung in der Ideenreihe stattfindet, welcher nur dem Heftschreiber zur Last gelegt werden kann. Was derselbe mit den Worten: „in einem Lande darf es nicht so seyn“ gemeint haben mag, ist rätselhaft, nur versichert der Herr Deponent, daß er keinen auch nur entfernt anstößigen Gedanken der Art jemals gehegt hat, noch jetzt hegt.

Was in den vorhergehenden Zeilen von Fortschritt und Mißverständnis gesagt ist, kann auf nichts anderes, als auf die Jugend, bezogen werden, scheint aber ebenfalls in seiner aphoristischen Gestalt nur dem Heftschreiber anzugehören. (ebd., B1. 201 f.)

Die ministerialen und universitären Zensoren beanstandeten auch andere Formulierungen im Heft des „Confilisters“ Carl von Willer. So im Punkt 4 die Formulierung vom „litterarisch-geheimen Bund“, im Punkt 5 eine Äusserung des Critical Review über Jakob I, im Punkt 6 die obsoleten Namen Filangieri oder Beccaria (,als die besseren Vorläufer einer schlechten Nachkommenschaft" - der französischen Revolutionäre), im Punkte 7 „das Emporkommen der Pfafferey in Spanien“, im Punkt 8 Über die Notwendigkeit bei dem Studium der Litteraturgeschichte auch die Staatengeschichte notwendig ist, im Punkt 9 wird der „Engel der Zweitracht“ von Wachler als Napoleon gedeutet, keinesfalls etwa der erzreaktionäre Metternich, im Punkt 10 wird die Wendung ,allerweltsklugheit“ beanstandet, ganz zu schweigen von einer durchaus revolutionären Wendung wie „das Ungethüm, welchem Widerstand geleistet werden muß“. Der Punkt 11 ,wegen der Mädchen-Erziehung“ und 12 S. 90 wegen „Bouterwek durften aus der im Eingange angeführten Entschuldigung 
milder zu beurtheilen seyn und soll in Ansehung Bouterweks auch angeführt werden, daß er durch seine Leidenschaftlichkeit gegen August Wilhelm Schlegel zu Repressalien Veranlassung gegeben“(ebd.).

\section{Ludwig Wachler als „Litteraturhistoriker“6}

Der von Wachler gebrauchte Terminus „Litteraturgeschichte“ bezieht sich in erster Linie auf die „Fachgeschichte“, wie sie sich in der jeweils aktuellen Forschungsliteratur zur geschichtlichen Entwicklung widerspiegelt. Anders ist es schon mit dem Begriff „Geschichte der teutschen Nationalliteratur“ oder auch mit dem Begriff der „NationalLiteratur" in Bezug auf die Literaturen einzelner europäischer und außereuropäischer Nationen. Hier meint Wachler nicht nur die schöngeistigen „National-Litteraturen“ samt literarischer Kritik, Philosophie, Gelehrsamkeit, Sprache, sondern auch, im zweiten Band seines Handbuches, die „Geschichte der Gelehrsamkeit ${ }^{\text {‘2 }}$. Diese Begriffsbestimmungen sind insofern wichtig, als man Wachler nicht unbedingt in einer nationalistischen Einengung des Jahnschen Turner-Patriotismus wahrnehmen soll, sondern als einen Gelehrten, der sich darum bemüht, zwischen einem nationalen und einem internationalen Anspruch ein Gleichgewicht der gegenseitigen Beeinflussung zu vermitteln. Die Vorträge zur deutschen Nationalliteratur hielt Wachler in Breslau vor 1819 (dem Jahr der Turnfehde und der Karlsbader Beschlüsse), nach 1819 immer seltener. So im WS 1824 Geschichte der Europäischen Nationallitteratur seit dem 16. Jahrhundert (Universität Breslau, 1824, S. VI), im SS 1827 wird die Vorlesung wiederholt. Im SS 1828 wird die Literaturgeschichte der neueren Zeit bereits von Karl Ludwig Kannegießer gelesen. Man darf also die vorsichtige Vermutung aufstellen, dass der Höhepunkt der Wachlerschen Tätigkeit im Bereich der Geschichte der „teutschen“ Nationalliteratur auf die Jahre 1815-1820 fällt. Die Veröffentlichungsdaten der beiden Bände sind hier von Bedeutung: 1818 für die Vorlesungen über die Geschichte der teutschen Nationallitteratur und 1824 für das Handbuch der Geschichte der Litteratur.

\section{Ziele, ideologischer Hintergrund, methodischer Standpunkt in den Vorlesungen über die Geschichte der teutschen Nationallitteratur}

Wachlers Stellung als Literaturhistoriker in Breslau war einerseits schwierig, weil er stets mit den ,germanistisch“ ausgebildeten Kollegen zu tun hatte: einerseits

\footnotetext{
${ }^{22}$ Dazu siehe die um 1800 ausgetragenen Diskussionen zum Begriff „Literatur“: „Was man im Deutschen gewöhnlich Litteratur nennt, ist Bücherkenntniß, oft nur Bücher-Titel-Kenntniß“ (Bouterwek, 1803, S. 131). Für die Belletristik und Poesie gab es zwei Begriffe: „schöne Litteratur“ und „schöne Wissenschaften“ (ebd., S. 132).
} 
mit von der Hagen, andererseits mit Hoffmann von Fallersleben. Spannungen im Verhältnis zu dem anderen sind durch Hoffmanns Zeugnisse belegt. Auf der anderen Seite waren seine Bemühungen -,,hochverdienstliche Vielthätigkeit“ (L. Wachler, 1818-1819, 1. Bd., S. VI) - insbesondere im Bereich der mittelalterlichen Literatur genügend dilettantisch, dass sich die genannten Kollegen nicht bedroht fühlten, zumal sich Wachler eindeutig auf die neuere und neueste Literatur konzentrierte, indem er nicht nur die Rolle eines Literaturhistorikers, sondern die eines Literaturkritikers zu spielen beabsichtigte. Insofern ist die „moderne“ Haltung Wachlers zumindest bis 1819 auch in dieser Hinsicht nicht zu übersehen ${ }^{23}$. Seine Vorlesungen, die er gleich nach seinem Antritt in Breslau hielt, waren für die Buchveröffentlichung ,vielfach umgearbeitet“ worden, sie sind auch nicht als eine komplette wissenschaftliche Darstellung gedacht, sondern eher als Anregung, „die Teilnahme an unserer vaterländischen Litteratur" (L. Wachler, 1818-1819, 1. Bd., S. V) zu wecken. Das Ziel ist aber nicht in erster Linie ein interessenloses Kennenlernen der Litteratur - dieses wurde von Wachler als ,das müßige bedeutungslose Spiel mit literarischen Alterthümern und Seltenheiten" abgetan (ebd., S. 1) -, sondern die Bekräftigung des Vaterlandes und des Volkes, und zwar in drei historischen Dimensionen: Die „Betrachtung über teutsches Kunstleben in Sprache und Schrift“ ist

\begin{abstract}
mehr als alles andere geeignet, vaterländischen Geist zu erwecken und zu erkräftigen, greifet tief und fast unmittelbar in das Bildungswesen des jugendlichen Geistes ein, befruchtet das Gemüth der Erwachsenen mit heimathlichen Bildern und Vorstellungen, verschmizet Wirklichkeit und Sehnsucht nach dem Höheren, adelt die Gegenwart, sie versöhnend mit der in ihren Bestrebungen und Freudegenüßen verjüngt aufblühenden Vergangenheit, und schließet das Geheimniß froher Zukunft auf, indem die Einbildungskraft, durch Geist und Gefühl der Vorzeit genährt, für sittliches Wollen und Streben frische Lebenskraft gewinnt. (ebd., S. 1)
\end{abstract}

Die drei Schritte der geschichtlichen Entwicklung sind bereits dem romantischen Denken verpflichtet, indem sie die philosophisch-universalistischen Kategorien der Jenaer Romantiker in den Bereich der Gemeinschaftsbildung und gleichzeitig der ethischen individuellen Verpflichtung übertragen. Nicht umsonst werden hier die Entwicklungsstufen des Individuums mit den Entwicklungen des Volkes in

\footnotetext{
${ }^{23}$ Hier verweise ich auf die drei Kampf-Broschüren Wachlers: „Freymüthige Worte über die allerneueste teutsche Litteratur" (1817-1819). Wachler präsentierte seine Betrachtungen für die Breslauer Philomathie (im ersten Band) und aufgrund der Leipziger Messekataloge. Er beschäftigt sich zwar mit der Ganzheit des deutschen Schrifttums, widmet aber der schönen Literatur ziemlich viel Platz. Beachtenswert sind die bereits im 1. Band erfolgte Hervorhebung Goethes und Theodor Körners (1817-1819, I, S. 22) sowie vernichtende Bemerkungen im 3. Band über das Wochenblatt von Kotzebue: „Viele, - und das sind die Unschuldigen, welche sich, Gott besser's! durch Bezeichnung mit dem Namen der Neutralen geschmeichelt fühlen, schriftstellerisch leicht zu bedienen sind und gerne so wohlfeil wie möglich für ihr schwächliches Gemüth und für ihre durch Essen, Spielen und Nichtstun sehr beschränkte Zeit von Allerley lesen und sprechen mögen, finden darin bequemen Zeitvertreib und leicht verdauliche Nahrung“(1817-1819, III, S. 13), dies am Vorabend des Breslauer Turnstreites.
} 
Parallele gebracht. Die „Kenntniß seines [des deutschen Volkes] Lebens in Wissenschaft und Kunst“ (ebd., S.5) sei nun die „bürgerliche Pflicht“, die sich in der „öffentlichen Meinung“ zu äußern hat. Diese demokratisch-ethische Prämisse des Patriotismus $^{24}$ lebt von dem Schema einer Befreiung aus den Ketten der (französischen) Knechtung, das nationale Bewusstsein in Kunst und Wissenschaft war eine der wirksamen Ursachen für die „Errettung aus unwürdigen Banden“ (ebd.). Diese Rückbesinnung ist religiösen Charakters, denn „,es war Gottes Stimme, die das teutsche Volk in sein Inneres zurückwies“ (ebd., S. 4). Die Aufgabe des „Litteraturhistorikers" ist also - synchron gesehen - die Eigentümlichkeit des Volkes zu erforschen, „die Teutschheit an sich“ (ebd., S. 12) ${ }^{25}$, bei gleichzeitiger Berücksichtigung der diachronen Entwicklungen sowie derjenigen Unterschiede, die aus „Oertlichkeit und Zeit" resultieren. Bei der Behandlung der Literaturgeschichte sind also mehrere Gesichtspunkte notwendig (ebd., S. 10). Und einer dieser Gesichtspunkte ist die „Kette“, die den europäischen Ursprung innerhalb der griechischen Kultur, die alles Fremde in sich aufnahm und in „bezaubernder Selbständigkeit“ umgestaltete, mit „unserer Zeit“ - gerade der Zeit der Blüte des deutschen Volkes und der deutschen Literatur, verkörpert in Goethe, verbindet. Das Wesen des deutschen Volkes möchte also Wachler auch in der Synthese des klassisch-antiken Geistes mit den deutschen Traditionen erblicken und sie vor dem vergleichenden Hintergrund ähnlicher kultursynthetisierender Erscheinungen in „Italien, Spanien und England“ wahrnehmen. Nun ist sich Wachler dessen bewusst, dass eine direkte Rückkehr zu den alten Tugenden keinesfalls einen Regress bedeuten darf: es gilt, bei Rückbesinnung auf die sittlichen Errungenschaften der religiös-volkhaften Entwicklung ein künftiges Werk der Vereinigung von allen sozialen Schichten des Volkes in die Wege zu leiten, also eine nationaldemokratische Gesinnung zu schaffen:

Es werden die Kunstwerke in teutscher Sprache Einen Geist offenbaren, sich zu Einem Ganzen vervollständigen, in welchem für das teutsche Volk, nach allen seinen Bestandtheilen, die hervorstechenden Anschauungen seiner Welt und seines Lebens niedergelegt sind. (ebd., S. 20)

Die notwendige Europäisierung der tdeutschen Nationalliteratur versucht er in seinen Vorlesungen mit Hilfe des germanischen einheitsschaffenden Mythos zu untermauern:

Wo Germanen gesiegt und gelebt haben, in Spanien und Frankreich, in England und Italien thut sich Kunde auf von ihrer äußeren und inneren Geschichte, sind Spuren ihres Volksgeistes erkenn-

\footnotetext{
${ }^{24}$ In der älteren Forschungsliteratur wurde dieser Patriotismus partout als Nationalismus bezeichnet: Braunbehrens, 1974; Weimar, 1989, S.254-291. Über Wachler heißt es: „kann man von literaturhistorischem Nationalismus sprechen, ihn als Ersatzreligion (man denke an Wachlers Pathos der biblischen Sprache) und die zugehörigen Literaturgeschichten als Erbauungsbücher bezeichnen“ (ebd., S. 291).

${ }^{25}$ Sehr deutlich hört man das etwa in folgender Formulierung: „So ist der Teutsche unter den verschiedenartigsten Einwirkungen äusserer Zeiumstände mehr geblieben, als geworden“(ebd.).
} 
bar, begegnen verwandte Vorstellungen, Bilder und Sagen, oft die Erstlinge des zwischen Rhein und Donau sich gestaltenden geistigen Kreises. (ebd., S. 22)

Die germanische Hypothese für eine kulturelle Einheit Europas war insbesondere nach der Niederlage Preußens 1806 gang und gäbe, meistens mit einem starken antifranzösischen Affekt gepaart, wobei man aus den hier geäußerten Bemerkungen Wachlers nicht unbedingt auf einen rigiden Nationalismus und Rassismus schließen darf. Einer der Vorläufer seiner Vorlesungen war Adam Müller, „geistreicher, tiefer, bindender für Schönheitssinn“ (ebd., S. 23), dessen in Dresden gehaltenen Vorträge (Müller, 1807) Wachler stark inspirierten. Gerade Müller hat die von Wachler akzeptierte romantische Überzeugung artikuliert, dass ,das Wesen deutscher Schrift und Kunst" sich keinesfalls in einem einzelnen Schriftsteller, ,vielmehr nur im Ganzen“ (ebd., „Programm“, [S. 1]) manifestiere. Es gab freilich einen markanten Unterschied zwischen den beiden: Müller urteilte aus der Perspektive der Niederlage, Wachler aus derjenigen des Sieges. War der Siegesberauschte in seinen Breslauer Vorlesungen zur nationalen Ausschließlichkeit geneigt, so plädierte der romantische Theoretiker für eine „passive“, jeder Aggression bare „Universalität“ (ebd., S. 3) der deutschen Kultur, die er als ein Gesetz jeder kulturellen Entwicklung anerkennt: „Jede Nation will ihren eignen Charakter, ihre eigne Ansicht vor allen Dingen den übrigen mittheilen“, ein Mittel dazu ist „die anderen Nationen mit Respect vor ihrer Individualität zu erkennen und fortdauernd zu beobachten“ (ebd., S. 10). Das große Verdienst des romantischen Denkens in Bezug auf den sich noch im Fließen befindlichen Begriff „Litteratur“ ist wohl ein ganzheitlicher Anspruch, der sich in der Überzeugung bemerkbar machte, dass ,,die politische oder die ökonomische und die poetische Existenz einander beständig bedingen“" (ebd., S. 161). Der Anspruch der Romantiker, das Volk bei ihren Darstellungen und Forschungen zu berücksichtigen, führte auch bei Wachler postulativ zur Berücksichtigung des politischen und sozialen Hintergrundes der literarischen Entwicklung, was ich anhand seiner Darstellung zur Poesie des Mittelalters, des Barocks und des 18. Jahrhunderts demonstrieren möchte.

Die Entdeckung des Mittelalters im 18. und zu Anfang des 19. Jahrhunderts gehört wohl zu den wichtigsten Ursachen für die Entstehung der universitären Germanistik. Auf der einen Seite konnte man die Vielfalt der dichterischen Formen in ihrer Adaptation durch den deutschen (oder germanischen) Geist demonstrieren, zum anderen glaubte man die ursprünglichste Emanation der "teutschen“ Volkseele gefunden zu haben, vor allem in Bezug auf das vermeintlich ursprünglichste Epos der mittelalterlichen Literatur, das Nibelungenlied. Auf jeden Fall ist die Wachlersche Perspektive modern, d. h. er erwähnt stets die Bemühungen der Schriftsteller und Wissenschaftler, Schätze der mittelalterlichen Literatur zu entdecken, zu edieren und volkstümlich zu machen: „unser Zeitalter mag stolz darauf seyn, daß dieses Kleinod in ihm bekannter, richtiger gewürdigt, vielseitig untersucht und als Eckstein 
volksthümlicher Kunst anerkannt, in Unterrichtsanstalten eingeführt (...) worden ist" (L. Wachler, 1818-1819, 1. Bd., S. 85). In dem Sinne ist das Wachlersche Denken erstens durch ein finales Konzept einer volkstümlichen Literatur geprägt (dazu gehört nicht nur das Nibelungenlied, sondern im noch größeren Maße das sogenannte „Heldenbuch“'26. Wachler verfährt dabei normativ im Hinblick auf die rein deutsche oder germanische Volkstümlichkeit der Dichtung, verschweigt aber keineswegs die kulturellen Übergänge zwischen der Provence, Spanien, der Bretagne und England. Er ist durchaus imstande, den künstlerischen Wert der Dichtungen zumindest verbal zum Ausdruck zu bringen, und immer wieder verweist er auf die neueren und neuesten Forschungen sowie dichterische Umarbeitungen der mittelalterlichen Stoffe (z. B. Ludwig Tieck, Minnelieder aus dem schwäbischen Zeitalter, Berlin 1805). In dem Sinne ist er mehr ein Wissenschafts- als Literaturhistoriker, und so reflektiert er viel stärker seine eigene Zeit als die Epoche des Mittelalters, an die er die Kriterien einer Volkstümlichkeit des 19. Jahrhunderts anlegt. Besonders werden von ihm zwei der Breslauer Frühgermanisten genannt: von der Hagen und J. G. G. Büsching, die ihm einiges mündlich über ihre Projekte mitgeteilt haben dürften ${ }^{27}$. Selbstverständlich waren Urteile Wachlers aus zweiter Hand geschöpft, man spürt hier deutlich den Einfluss der beiden genannten Breslauer Ordinarien. Allerdings ist seine wissenschaftsgeschichtliche Positionierung der Forschungen zum Mittelalter nicht ganz von der Hand zu weisen. Ähnliches gilt auch für seine Darstellung einer damals so problematischen Epoche wie der Barock des 17. Jahrhunderts.

So stellte Klaus Garber fest, dass das Postulat der Volkszugehörigkeit ,potentiell ein egalitär-demokratisches Moment gegenüber der ständischen und dynastischen Spaltung des spätabsolutistischen Staates“ (1976, S. 103 f.) sei. Bei Wachler und vielen anderen „Turnern“ verschwimmt freilich „die Kraft der Negation dieses

${ }^{26}$ „Unter dem Heldenbuche wird eine Blumenlese teutscher Helden-Sagen von uralter Grundlage und verschiedener Bearbeitung verstanden“ (L. Wachler, 1818-1819, Bd. 1, S. 82). Es handelt sich um ein Projekt, dass von der Hagen verwirklichen sollte (von der Hagen \& Primisser, 1825; vgl. Haustein, 1989).

${ }^{27}$ Wachler erwähnt von der Hagen mit der Edition niederdeutscher Psalmen 1816 (1818-1819, 1. Bd., S. 40 sowie von der Hagen und Büsching im Zusammenhang mit der Geschichte von Herzog Ernst von Bayern von Heinrich von Veldegk (ebd., S. 56), er berichtet über von der Hagens Ausgabe des Werkes von Heinrich von Friedberg (ebd., S. 75), lobt Büschings und v. d. Hagens Gedichte des Mittelalters, Berlin 1808, (ebd., S. 78) und würdigt nicht zuletzt die von der Hagenschen Ausgaben des Nibelungenliedes: „Diesen Abdruck ließ F. G. [sic] von der Hagen sorgfältiger und nicht ohne Berichtigungen (Berlin 1810) wiederholen; in einer zweyten Ausgabe (Breslau 1816 gr. 8), welcher bald eine dritte vielfach kritisch berichtigte folgen wird, wurde die S. Galler Handschrift zu Grunde gelegt“ (ebd., S. 92; diese dritte Ausgabe ist als die „Zweite umgearbeitete Ausgabe“ in Frankfurt am Main 1824 erschienen). Außerdem erwähnt Wachler noch das Hagen-Büschingsche ,altdeutsche Museum“, in dem Allegorien Conrads von Würzburg abgedruckt wurden (ebd. S. 100 f.) und Salomon und Morolf, das ,in dem ersten Bande der v. d. Hagen-Büsching`schen Sammlung“ abgedruckt wurde (ebd., S. 101). 
Begriffs, die ihm bei Herder und den Dichtern des Sturm- und Drangs innewohnte, in ahnungsloser Fabuliererei von der erträumten Einheit des Volksganzen" (ebd., S. 104). Es werden Wachler auch „schwammige Begriffe“"vorgeworfen, Garber drückt allerdings eine gewisse Achtung Wachler gegenüber aus, indem als Vorzug sieht Garber Wachlers Nichtbeachtung der Opitzschen Standespolitik, was zum „obligaten Vorwurf der liberal-freiheitlich gesinnten Literaturhistoriker, wie etwa Gervinus, gehörte“ (ebd., S. 108).

Wachlers Einschätzung geht vom „Volk“ aus und mündet auch im schwammigen Begriff „Volk“, der Gelehrte unterstreicht aber handfeste Ursachen für die Entwicklung der Poesie gerade im Schlesien des 17. Jahrhunderts. Er weist in erster Linie auf Bildungsphänomene hin: „So hatte sich ein Teil des Adels und des höheren Bürgerstandes festeres Selbstgefühl, Empfänglichkeit und Anspruch auf gesellschaftliche und geistige Bildung angeeignet" (L. Wachler, 1818-1819, 2. Bd., S. 19). Er betont die Entwicklung des Schulwesens im Schlesien des 16. Jahrhunderts - es wird zum Beispiel Melanchtons Schüler Valentin Friedland Trotzendorf erwähnt (ebd., S. 20) -, ein weiterer Faktor war für den Konsistorialrat die Reformation. Sie habe zwar „die Eintracht und das wechselseitige Vertrauen“ (ebd.) gestört, auf der anderen Seite begünstigte sie die erwähnten Bildungsanstrengungen der oberen Schichten, die allerdings in ,kleinen Kreisen, unter Gefahren und nothwendig gewordenen Anstrengungen“ (ebd., S. 19) erfolgten. In großen Gefahren (Krieg und die mögliche Rekatholisierung) haben sich „große Kräfte entwickelt“. Die Religionsstreitigkeiten betrafen nicht nur die Gelehrten, sondern auch „das Volk“, das zu „ernst-tiefem Nachdenken, zu fruchtbaren Betrachtungen, zur Uebung und Bewährung des frommen Gemeingeistes, zu beharrlichen Entschliessungen und Bestrebungen" Anlass fand. Durch diese religiöse Bindung kam auch das (schlesische) Volk in den Genuss der poetischen Entwicklung des Landes, was die zahllosen schlesischen Nachahmer Opitzens unter Beweis stellten: "Pfarrherren, Schulmänner, Küster, Universitätslehrer, Studenten und Schüler drängten sich zu diesem Berufe" (ebd., S. 29), so dass die Schar der Versemacher, insbesondere in Schlesien bald ,unglaublich groß“" war. Das Volk (offensichtlich im Sinne einer einheitlichen nationalen Gemeinschaft) und die protestantische Religion sind die beiden Fluchtpunkte in der literarhistorischen Reflexion Wachlers. Die katholischen Autoren der Epoche der Konfessionalisierung werden zwar marginal behandelt (Johannes Scheffler wird nur als Liederdichter erwähnt), solche Persönlichkeiten wie der in der Romantik entdeckte Abraham a Santa Klara (Urich Megerle; ebd., S. 96 f.), oder Friedrich von Spee werden aber durchaus positiv charakterisiert ${ }^{28}$. Die beiden großen Vertreter der

\footnotetext{
${ }^{28}$ Man muss betonen, dass bei den beiden Breslauer Professoren J. G. G. Büsching und Ludwig Wachler die Abneigung gegenüber dem Katholizismus sogar ihre Einschätzung der in der Zentralbibliothek versammelten Handschriften als „Kloster-Unrat“ beeinflusste. Bei Büsching lesen wir: „Dann ärgere ich mich über das verdammte Nonnenvolk. Ich glaube gewiss, mir zum Ruhme nachsagen zu
} 
sogenannten zweiten schlesischen Schule werden von Wachler in Bausch und Bogen verdammt; Caspar Lohenstein und Christian Hoffmann von Hoffmannswaldau vereinigen mit dem „unteutschen Prunke“ „teutsche Pedanterey“ (ebd., S. 76). Eine Ausnahme wird für Arminius und Thusnelda, eine Heldengeschichte Lohensteins, gemacht: ,nicht allein die Sprache ist oft vortrefflich, sondern auch in geschichtlichen Schilderungen und in Reden kann kein Zeitgenosse mit Lohenstein verglichen werden, ohne sehr zu verlieren“ (ebd., S. 78).

Ganz apologetisch ist die Wachlersche Bewertung der Dichter des 18. und 19. Jahrhunderts. Goethe ist für ihn der „größte unserer Dichter“ (ebd., S. 291). Die Größe resultiert aus dem Verständnis der Epoche: „Göthe gehört seiner Zeit an; er versteht sie und spricht sie aus" (ebd., S. 292). Das höchste Lob wird mit dem Votum „Göthes Werk gehört dem teutschen Volke an“ (ebd., S. 293) formuliert. Das auf der vorletzten Seite der Goethe-Vorlesung geäußerte Lob der Romantiker ${ }^{29}$ (Ludwig Tieck, Novalis, Heinrich von Kleist, Friedrich de la Motte Fouqué) ist eine pauschale Huldigung der neuen Strömung, die vor allem ethischen Charakters ist: „das gute Alte wird hervorgesucht, das Treffliche in seiner schönen Tiefe erfaßt; der teutsche Sinn im Kunstgenusse gewinnt täglich an Lebendigkeit und Kraft" (ebd., S. 308 f.). Dass Wachler den Polemiken seiner Zeit fern stand und seine Urteile aus der Perspektive eines Unbeteiligten fällte, belegt die Reaktion der Jungdeutschen auf seinen Goethe-Kultus. Er wurde kritisch vermerkt vor allem im Menzels „LiteraturBlatt zum Morgenblatt für gebildete Stände“, allerdings in Bezug auf ein anderes Werk Wachlers, nämlich auf das in der zweiten Auflage erschienene Lehrbuch der Litteraturgeschichte (Leipzig, Barth 1830):

Was ferner Goethe betrifft, so hat zwar Wachler dessen mittlere und höhere Stellung zwischen und über jenen beiden Schulen des Scherzes und Ernstes, oder der Spielerei und Pedanterei, sehr richtig bezeichnet, wenn er aber im Schlußsatz die ganze fernere Entwicklung der deutschen Literatur, selbst der gelehrten, in Verbindung mit dem Lobe Goethes bringt, so ist diese Zusammenstellung wohl etwas schmeichelhaft. (G[utzkow], 1832, S. 399)

Ähnlich kritisch hat sich Wolfgang Menzel (1833, S. 405) zur dritten Umarbeitung des Handbuches der Geschichte der Literatur, Leipzig, Barth 1833 geäußert.

können, dass ich mit grosser Liberalität und Humanität verfahre; ich habe ihnen, besonders der Frau Subpriorin, grade der Schwätzerin, ganz ohne dazu authorisirt zu sein, auf meine eigene Gefahr, so dass ich noch nicht weiss, ob ich nicht darüber verantwortlich sein werde, eine bedeutende Menge Gemälde, Königlichen Eigenthum, geschenkt und dann schwätzt das Volk so infam hinter dem Rücken.- Wäre ich so mit Leib und Seele ein Berliner, dann würde ich mir nichts daraus machen, oder - doch ich mag über das Zeug nicht mehr reden. - Ausserdem habe ich durch Klatscherei, andere Dinge erfahren, die mir sehr unangenehm sind und die machen werden, dass ich bald hier in offenbaren Kampf trete; denn wenn sie mit ihren Lumpereien mir in den Weg treten, so halte ich mich nicht stille, es mag dann gehen, wie es will.“ (Żarski \& Żarska, 2017, im Druck).

${ }^{29}$ Die Bezeichung ,romantisch“ bezog sich für Wachler, aber auch für Adam Müller auf die ritterlichen Epen des Mittelalters (L. Wachler, 1818-1819, Bd. 1, S. 65, 6. Vorlesung: „Das romantische Epos“). 


\section{Versuch einer europäischen Nationalliteratur? Texte und Kontexte}

Die erste Ausgabe des Handbuches erschien $1805^{30}$ als Handbuch der allgemeinen Geschichte der literärischen Cultur von D[r]. Ludwig Wachler, Consistorialrathe und Professor der Theologie und der historischen Wissenschaften zu Marburg. Zweyte Hälfte. Geschichte der neueren Zeit. Marburg, in der neuen akademischen Buchhandlung 1805. Die zweite Ausgabe trug bereits einen geänderten Titel, und zwar: Handbuch der Geschichte der Litteratur von Dr. Ludwig Wachler. Zweyte Umarbeitung. Dritter Theil. Geschichte der neueren Nationallitteratur. Frankfurt am Main, Verlag der Hermannschen Buchhandlung 1824. Die dritte, oben erwähnte Ausgabe erschien als Handbuch der Geschichte der Litteratur von Dr. Ludwig Wachler. Dritte Umarbeitung. Dritter Theil. Geschichte der neueren Nationallitteratur. Leipzig, 1833. Verlag von Joh. Ambr. Barth. Wachler hat sein Unternehmen im Alleingang verwirklicht, was umso mehr verwundert, als wenig früher ein ähnliches Projekt an der Universität Göttingen startete, und zwar die Geschichte der Künste und Wissenschaften seit der Wiederherstellung derselben an das Ende des 18. Jahrhunderts. Von einer Gesellschaft gelehrter Männer ausgearbeitet. Als erster Band in der dritten „Abtheilung“ erschien dann Friedrich Bouterweks Geschichte der schönen Wissenschaften (Göttingen, bey Johann Friedrich Röwer) ${ }^{31}$. Es gab noch ein Vorgängerunternehmen, nämlich die von Johann Gottfried Eichhorn 1796-1799 verfasste und bei Johann Heinrich Rosenbusch in Göttingen erschienene Allgemeine Geschichte der Cultur und Litteratur des neueren Europa. Mit dem zweiten Band wurde das Werk in die oben erwähnte Geschichte der Künste und Wissenschaften seit der Wiederherstellung derselben eingeschlossen und lief so parallel zu dem 1805 veröffentlichten Werk Wachlers. Es gab freilich einen gravierenden methodischen Unterschied zwischen Wachler und seinen Göttinger Konkurrenten. Wie Bouterwek in der Vorrede zum ersten Band seiner Geschichte der schönen Wissenschaften betont:

Wer sich an die weitaussehende Unternehmung wagt, die Geschichte der neueren Poesie und Beredsamkeit zu erzählen, hat, wenn er nicht ein endloses Werk anfangen will, zwischen zwei Wegen zu wählen. Der eine ist der philologisch-bibliographische, der andre der philosophisch-kritische. (Bouterwek, 1801, Vorrede)

Bouterwek, wie bereits früher Eichhorn, hat den zweiten Weg, also den philosophisch-kritischen, gewählt, Wachler dagegen versuchte eine Mischung aus der philo-

\footnotetext{
${ }^{30}$ Der Vorgänger des Handbuches war der von 1793 bis 1801 in zwei Teilen erschienene Versuch einer allgemeinen Geschichte der Litteratur, der von Peter Goßens als Ausdruck der alten universalen Historia litteraria charakterisiert wird. In den weiteren, hier besprochenen Ausgaben des Handbuchs „nimmt er sowohl in der historischen als auch in der wissenschaftsspezifischen Darstellung der Neuzeit entscheidende Änderungen vor, die letztlich zur Etablierung der literaturhistorischen Gattung des 19. Jahrhunderts, also der allgemeinen bzw. der Weltliteraturgeschichte führen“" (Goßens, 2011, S. 37 f.).

${ }^{31}$ „Schöne Wissenschaften“ bedeutete damals „,schöne Literatur“.
} 
logisch-biographischen und philosophisch-kritischen Methode, was den Regeln eines modernen, auch heute benutzten Handbuches wohl besser entsprach. Eine andere Sache ist die Frage der narrativen Probleme. Wachler behandelt einzelne Nationalliteraturen getrennt, er betont zwar gegenseitige Beeinflussungen und Abhängigkeiten, verfährt aber nicht wie die Göttinger, die in der chronologischen Abfolge die gegenseitige Beeinflussung der Literaturen nach dem organologischen Prinzip der Wachstumsphasen, Blüteperioden und Spätphasen verfuhren und somit die Abfolge der Kulturen und Literaturen berücksichtigten, wobei die deutsche Literatur eine sehr spezifische Stellung hat, denn ,wer kann die Geschichte des deutschen Geschmacks in irgend einer Kunst erzählen, wenn er nicht den Geschmack von halb Europa als bekannt voraussetzt“ (ebd., S. 7). Von dieser frühen Prämisse, die eigentlich eine europäische Nationalliteratur konstruiert - im Sinne einer Literatur, die im „Volk“ angesiedelt sei -, ist es nur ein Schritt zu der romantischen Auffassung einer spezifischen Vermittlerrolle, die die deutsche Literatur und Kultur an Stelle der schweigenden bzw. vorläufig, weil nach einer entscheidenden Niederlage des Jahres 1806, schwachen Waffen zu spielen hat:

Nicht im Unterdrücken, sondern im höchsten Gedeihen der Bildung unsrer Nachbarn finden wir unser eignes Glück, und so wird Deutschland (das glückliche Mittelland), wenn es Europa durch seinen Geist beherrsche, nie seinen Gehorsam und seine Achtung gegen die übrigen zu verläugnen brauchen. (Müller, 1807, S. 11)

Auch im Handbuch wird der Begriff Nationalliteratur im Sinne von „schöne oder schöngeistige Literatur" verstanden, der Begriff der Literatur ohne Prädikat bezieht sich dagegen darauf, was Bouterwek als „wissenschaftliche Litteratur“ zu nennen geneigt war. Es ist interessant, dass Wachler in der ersten Aufgabe den modern anmutenden Begriff der „litterärischen Cultur“ gebraucht, der auch in den nächsten Bänden beibehalten wird, und zwar in Bezug auf die ,gesellschaftliche“, aber auch auf die „litterärische Cultur“. In seinen Vorlesungen gebraucht er auch den Begriff „europäische Nationalliteratur“ offensichtlich als Kollektivsingular, was zwei Deutungsmöglichkeiten zulässt. Die erste resultiert aus der Tatsache, dass er nicht imstande ist, die außereuropäischen Kulturen und Literaturen zu begreifen, deshalb lässt er sie schlichtweg links liegen („Die litt[erärische] Cultur der aussereuropäischen Völker ist von geringem Umfange (...)“ (L. Wachler, 1833, 3. Bd., S. 510) - dies trotz des West-Östlichen Divans! Im Begriff ,europäische Litteratur“" kann sich aber in positiver Hinsicht ein Bedeutungskeim verbergen, der auf eine Gemeinsamkeit von europäischen Literaturen schließen lässt, etwa eine parallele Prägung zur „Weltliteratur" Christoph Martin Wielands.

Imponierend ist nicht nur die Tatsache, dass Wachler die Nationalliteratur der kulturell kleinen Völker (das sind für ihn, gemessen am Umfang der Informationen, Polen, Schweden, Böhmen, Russen, Sorben, insgesamt die slawischen Völker) schlecht oder recht berücksichtigt und somit im Herderschen Sinne durchaus die 
nationale und kulturelle Vielfalt Europas anerkennt, sondern auch, dass er die Korrekturfähigkeit von eigenen Meinungen, und zwar unter dem Einfluss der geschichtlichen Ereignisse, unter Beweis stellt. Ist die Narration zur deutschen bzw. germanischen Literatur an den beiden Polen orientiert: den vermeintlich beständigen Charaktermerkmalen einerseits und einer Entwicklung zur Bildungsperfektibilität andererseits, so unterliegt die Erzählung über die polnische Kultur (immer nach dem Schema: Übersicht, Sprache, Poesie, Prosa) einem durch die protestantischfortschrittlich orientierte Pädagogik geprägten Schema der politischen Selbstverschuldung der Polen. Der Staat, zwischen Jesuiten und Magnaten zerrissen, mit dem geknechteten Volk und fehlendem Bürgerstand, wurde 1794 annihiliert zugunsten fortschrittlicher staatlicher Organismen, die ihrerseits einen Versuch der Reformen unternahmen, wie etwa die Preußen im Warschau des Jahres 1801 durch die Gründung einer Gesellschaft für Wissenschaften und Künste. Die Poesie ist zwar vielfältig, aber verdient kaum den Namen einer Nationalliteratur, weil sie überhaupt nicht mit dem „Volk“ verbunden war - so die durchaus in Deutschland gängige Narration in der ersten und der zweiten Fassung des Handbuches. Wesentliche Änderungen werden allerdings in der dritten Fassung des Handbuches von 1833 vorgenommen. Polen wurde erstens nicht nur durch das eigene Verschulden geteilt, sondern „durch feindseliges Zusammentreffen der unglücklichsten Zeitverhältnisse“ (L. Wachler, 1833, 3. Bd., S. 480) - so die freundliche Umschreibung für den russisch-preußischen Pakt und die Rolle Russlands und Preußens bei der Niederschlagung der von Wachler ziemlich breit und mit Sympathie [!] geschilderten Reformversuche: Gross war der Eifer (s[eit] 1760), welcher sich dieser hochwichtigen Angelegenheiten beurkundete;
Stan. Konarski (geb. 1700 gest. 1773) erwarb sich um das Erziehungswesen viele Verdienste; unter
den Grossen waren nicht wenige von begeisterter Liebe für Kunst und Wissenschaft beseelt, so der
gelehrte J. Andr. Zaluski (geb. 1701 st. 1773), Wenc. Rzewuski (geb. 1705 st. 1779), G. Ad. Czarto-
ryski (geb. 1733 st. 1823) u. m. a. Nach schmerzlichen Erfahrungen (1733) [wieder eine Umschrei-
bung für die erste Teilung Polens!] reifte (1791) der rasch zur Ausführung gebrachte Entwurf, eine
veste Verfassung und in dem Bürgerstande eine Nation zu begründen, und endete (D 10. Oct. 1794)
durch feindseliges Zusammentreffen der unglücklichen Zeitverhältnisse und des gehässigsten
Zwiespaltes politischer Meinungen mit der Auflösung des Staates. (ebd.)

Dass es sich um einen aufklärerisch verbrämten Landraub gehandelt hatte, wurde freilich mit keinem Wort erwähnt, die Wahrheit schimmert lediglich durch die sehr vorsichtigen Formulierungen, an denen auch die damalige Zensur ihren Anteil haben dürfte, hindurch. Diese Zunahme an Sympathie und vor allem an exaktem Interesse für polnische Kultur ist bei dem ehemals freiheitlich gesinnten Wachler bestimmt durch den Novemberaufstand ausgelöst worden: der Gelehrte tut zwar die Ereignisse der Novembernacht als „blutbefleckte Gewaltthaten“ (ebd., S. 481) ab, informiert aber den Leser über den fassadenartigen Charakter der vom Zaren dem sogenannten Königreich Polen verliehenen Verfassung (die „im todten Buchstaben“ bestand, wie „Viele glaubten“). Zum ersten Mal erwähnt Wachler auch neben einer 
reichen Vertretung der polnischen Literatur seit dem 16. Jahrhundert den ,geistreiche[n] Urheber der romantischen Poesie" Adam Mickiewicz und beruft sich auf seine 1829 in Paris veröffentlichten Schriften (ebd., S. 483). Wachler musste also umlernen und war, fast am Ende seines Lebens, dazu fähig, obwohl die Umstände ihm nicht erlaubten, Klartext zu reden. Auch in Bezug auf Russland und dessen Kultur machte Wachler bedeutende Fortschritte, indem er richtig prophezeite, dass sich die russische Literatur bald von den westeuropäischen Mustern emanzipieren werde. Die politischen Sympathien für das Zarenreich waren bei ihm ohnehin vorhanden.

Am 8. April 1838 benachrichtigte die Witwe den Rektor der Universität, dass ihr Gemahl ,am 4ten des Mts., morgens um drei Viertel auf Vier Uhr im fast vollendeten 71sten Lebensjahre, im 50ten Jahre amtlicher und litterarischer Wirksamkeit, im 49 Jahre seiner glücklichen Ehe" verschied ${ }^{32}$.

\section{Bibliographie}

\section{Literatur}

Bouterwek, F. (1801). Geschichte der schönen Wissenschaften, 1. Bd. Göttingen: Röwer

Bouterwek, F. (1803). Idee einer Litteratur. Neues Museum der Philosophie und Literatur, hrsg. von F. Bouterwek (S. 131-143). Leipzig: Martini.

Braunbehrens, V. (1974). Nationalbildung und Nationalliteratur. Zur Rezeption der Literatur des 17. Jahrhunderts von Gottsched bis Gervinus. Berlin: Spiess.

Garber, K. (1976). Martin Opitz, ,der Vater der deutschen Dichtung“. Eine kritische Studie zur Wissenschaftsgeschichte der Germanistik. Stuttgart: Metzler.

Gaß, W. (1878). Gaß, Joachim Christian. Allgemeine Deutsche Biographie (8, S. 394-396). Abgerufen unter https://www.deutsche-biographie.de/gnd116448539.html\#adbcontent.

Goßens, P. (2011). Weltiteratur. Modelle transnationaler Literaturwahrnehmung im 19. Jahrhundert. Stuttgart: Metzler.

G[utzkow, K.?] (1832). Lehrbuch der Literaturgeschichte von Dr. Ludwig Wachler. Morgenblatt für gebildete Stände. Literatur-Blatt (S. 397-399). Stuttgart: Cotta.

von der Hagen, F. H. \& Primisser, A. (Hrsg.). (1825). Der Helden Buch in der Ursprache, 2. Bd. Berlin: Reimer.

Haustein, J. (1989). Der Helden Buch. Zur Erforschung deutscher Dietrichepik im 18. und frühen 19. Jahrhundert. Tübingen: Niemeyer.

Johann Friedrich Ludwig Wachler. (1840). In Neuer Nekrolog der Deutschen (16. Jg. 1838, I. Theil), S. 361-373. Weimar: Voigt.

Löffler, R. (2011). Pädagogik, Politik, Wissenschaft: der Historiker Ludwig Wachler (1767-1838) in seiner Zeit. Frankfurt M.: Lang.

Markgraf, H. (1892). Gustv Adolf Harald Stenzel's Wirksamkeit und Bedeutung für die schlesische Geschichtsschreibung. Vortrag zur Erinnerung an seinen hundertjährigen Geburtstag. Zeitschrift des Vereins für Geschichte und Alterthum Schlesiens, 26, 395-447.

\footnotetext{
${ }^{32}$ GStAPK IHA Rep. 76 Va Sekt 4 Tit IV Nr. 10, Bd. 5. B1. 82 r.
} 
[Menzel, W.] (1833). Handbuch der Geschichte der Literatur, von Dr. Ludwig Wachler. Morgenblatt für gebildete Stände. Literatur-Blatt (S. 405-407). Stuttgart: Cotta.

Menzel, W. (1877). Denkwürdigkeiten. Bielefeld: Velhagen \& Klasing.

Müller, A. H. (1807). Vorlesungen über die deutsche Wissenschaft und Literatur (2., vermehrte und verbesserte Auflage). Dresden: Arnold.

Nowack, K. G. (1838). Schlesisches Schriftsteller-Lexikon, 2. Heft. Breslau: Korn.

Passow, F. (1818). Turnziel. Turnfreunden und Turnfeinden. Breslau: Max.

Rosenberg, R. (2009). Die deutschen Germanisten. Ein Versuch über den Habitus. Bielefeld: Aisthesis.

Rosenkranz, K. (1832). Geschichte der orientalischen und griechischen Poesie (Handbuch einer allgemeinen Geschichte der Poesie, Erster Theil). Halle: Eduard Anton.

Universität Breslau (Hrsg.). (1824). Verzeichniß der auf der Universität zu Breslau, im Winter-Semester vom 18. Oktober 1824 an, zu haltenden Vorlesungen.

Wachler, A. (1838) Dr. Ludwig Wachler. Schlesische Provinzialblätter, Bd. 107, S. 405-418 Breslau.

Wachler. A. (Hrsg.). (1839). Franz Passow's Leben und Briefe. Breslau: Hirt.

Wachler, L. (1802). Aphorismen über die Universitäten und über ihr Verhältniss zum Staate. Nebst einem Anhange über den gegenwärtigen Zustand der Universität zu Marburg. Marburg: in der neuen akademischen Buchhandlung.

Wachler, L. (Hrsg). (1812). Friedrich Wilhelm Strieder's Grundlage zu einer hessischen Gelehrten- und Schriftstellergeschichte. Von der Reformation bis 1806, 16. Bd. (Steuber-von dem Werder). Marburg: Akademische Buchhandlung.

Wachler, L. (1814). Worte vaterländischer Hoffnung. Den edeln und biederen Männern teutscher Nation an das Herz gelegt. Marburg: Kriegler.

Wachler, L. (1817-1819). Freymüthige Worte über die allerneueste teutsche Litteratur, 3 Bde. Breslau: Holäufer.

Wachler, L. (1818-1819). Vorlesungen über die Geschichte der teutschen Nationallitteratur, 2 Bde. Frankfurt: Hermann.

Wachler, L. (1833). Handbuch der Geschichte der Litteratur, 4 Bde. Leipzig: Barth.

Wachler, L. (1835). Biographische Aufsätze (Vermischte Schriften, Bd. 1). Leipzig: Barth.

Wägenbauer, B. (2000). Georg Friedrich Benecke (1762-1844). In C. König, H.-H. Müller \& W. Röcke (Hrsg.), Wissenschaftsgeschichte der Germanistik in Porträts (S. 6). Berlin: Gruyter.

Weimar, K. (1989). Geschichte der deutschen Literaturwissenschaft bis zum Ende des 19, Jahrhunderts. München: Fink.

Żarski, K \& Żarska, N. (Hrsg). (2017). „Die Schlesier im Ganzen taugen wahrlich nichts!“ Johann Gustav Gottlieb Büschings Briefe an seine Braut. An der Wiege der Breslauer Germanistik. Leipzig: Leipziger Universitätsverlag (im Druck).

\section{Archivalien}

(1) Archiv: Geheimes Staatsarchiv Preußischer Kulturbesitz. Berlin Bestand: Kultusministerium

GStAPK IHA Rep 76 Va Sekt 4 Tit X Nr. 1, Bd. 3, Bl. 184 r. (19. Februar 1815). [Schreiben des Innenministers Schuckmann an die Academische Verwaltungscommission in Breslau].

GStAPK IHA Rep 76 Va Sekt 4 Tit X Nr. 1, Bd. 3, Bl. 198 r. (9. März 1815). [Schreiben Wachlers an das Ministerium Altenstein].

GStAPK IHA Rep 76 Va Sekt 4 Tit X Nr. 1, Bd. 3. Bl. 185 r-186 v. (23. März 1815). [Schreiben des Ministeriums an Ludwig Wachler].

GStAPK IHA Rep 76 Vf Lit. W. Nr 7 Wachler, B1. 198. (1824). [Schreiben des Regierungsbevollmächtigten an das Ministerium]. 
GStAPK IHA Rep. 101 Nr. E Lit. A 06. ( o.J.) [Schreiben des Ministers Altenstein an den Innenminister Schuckmann].

GStAPK IHA Rep. 76 Sekt 4. Tit IV, Nr. 1, Bd. 5, Bl. 33r. (o.J.).

GStAPK IHA Rep. 76 Va Sekt 4 Tit IV Nr. 10, Bd. 5. Bl. 82 r. (8. April 1838). [Schreiben der Witwe Wachler an den Rektor der Universität in Breslau].

GStAPK IHA Rep. 76 Va Sekt 4. Tit. IV Nr. 1, Bd. 15, Bl. 15 r. (14. September 1829). [Schreiben des Universitätskurators Neumann an das Ministerium].

GStAPK IHA Rep. 76, Sekt 4, Tit IV Nr. 5. B1. 16 v. (4. November 1824). [Schreiben des Regierungsbevollmächtigten (Neumann) an Minister von Altenstein].

GStAPK IHA Rep. 76, Sekt 4, Tit IV Nr. 5. B1. 45 v-46 r. (9. Dezember 1822). [Schreiben des Ministers Altenstein an den Innenminister Schuckmann].

(2) Archiv: Hessisches Staatsarchiv Marburg Bestand: Nachlass L. Wachler

HStaM Nachlass L. Wachler. Brief des Ministers Altenstein vom 18. Juni 1819.

(3) Universitätsbibliothek Breslau, Handschriftenabteilung

AKC 1952 KN 9. (18 März 1814). [Brief von L. Wachler an den Staatsminister von Hardenberg, Marburg].

Aut. 1590. (17. 5. 1815). [Brief Wachlers Johann Samuel Ersch]. 